\section{TETANY AS A SEQUELA OF PUERPERAL ECLAMPSIA.}

BY S. W. WHEATON, M.D., M.R.C.P. LOND., D.P.H., PHYSICAA TO THE KOYAL HOSPITAL FOR CHILDREX AND WOMEN WATERLOO-ROAN, S.E.

Mrs. M- aged twenty, primipara, was confined at 10.45 A. M. on March 2nd, 1890. The labour was not unduly prolonged, the presentation was of the vertex, the child (a male) was lifing and there was no undue loss of blood. Before labour the patient was noticed to be pale and comjlained of pain in the loins; but as there was no oedema : nywhere the urine was not examined. Immediately after the expulsion of the placenta at 11 A.M. she was seized with convulsions. When I saw her for the first time at 3.15 P.Mr. thirteen attacks of general convulsions had occurred and she was lying in a comatose condition, with dilated pupils. The conjunctiral reflex was absent, respiration was rapid and irregular and crepitations were heard at the bases of both lungs; the temperature was $100^{\circ}$; the pulse was 150 , very feeble but incompressible. The secretion of urine was very scanty ; a few drachms drawn off by catheter were solid with albumen and contained blood, blood casts, and only $\frac{1}{2}$ per cent. of urea on subsequent analysis. I at once applied wet cupping to both loins and removed half a pint of blood in this way. An eighth of a grain of hydrochlorate of pilocarpin was injected subcutaneously, and twenty grains of chloral and one drachm of bromide of potassium were given per rectum. At 3.45 P.M. another convulsion of a typical epileptic character occurred, preceded by a sighing sound, and, what was particularly noticeable, by marked contraction of the uterus, which could be felt to project forward and become hard under the hand placed upon the abdomen. At 4 P.M. wet cupping and the pilocarpin injection were repeated ; from this time improvement commenced and profuse diaphoresis occurred. The next fit did not occur until 5.15 P. M., after which only three fits occurred until the next morning (March 3rd) at $4 \Lambda$. M., when two occurred within a short time of one another. From this time they ceased altogether. At 10 A.m. she was still unconscious, but could swallow ; the conjunctival reflex had returned and the temperature had risen to $103^{\circ}$. Dr. W. O. Priestley kindly saw her for me, and recommended liquor ammoniæ acetatis (two drachns every two hours), which was given, as also were a purge and enema. Thirty ounces of urine were collected during the twenty-four hours of this day, containing 4 per cent of urea and $\frac{1}{8}$ per cent. of albumen. The next day (March 4th) the patient continued unconscious and the temperature was continuously high at $102^{\circ}$ to $103^{\circ}$. There were no sions of lung mischief, but a systolic murnur appeared at the apex of the heart and also at the left base, but without increase of dulness in the cardiac area. Thirty-nine ounces of urine were drawn off.March 5th: This morning, while still unconscions, she was noticed by the nurse to have spasmodic attacks of rigidity of the limbs. When seen two hours later she was lying on her back with the arms rigidly extended by her sides, the hands semiflexed at the wrist, the proximal joints of the fingers of both hands flexed and the two distal joints extended ; the fingers were approximated and adducted as a whole and the thumbs were rigidly flexed into the palms. The legs were rigidly straightened out, the anklejoints were extended and the toes were adducted. The jaws were rigid, but not so much as to prevent the giving of food. Pressure on the limbs caused increased rigidity. This condition, which was thus typical of tetany, lasted for three days, at the end of which time it gradually disappeared, consciousness also returning slowly. The temperature, which had continued at $102^{\circ}$ to $103^{\circ}$, became normal, and the urine increased in quantity to fifty-two ounces. There was complete loss of memory regarding the events preceding the fits, and for some time the patient would not believe that she had been confined. Secretion of milk was entirely absent, but the involution of the uterus proceeded normally. In three weeks convalescence was complete; there was only a trace of albumen in the urine and the cardiac murmurs had disappeared. Six months later the urine contained a trace of albumen. I have seen her twice since, the last time three months ago, and on each occasion albumen has been absent from the uine and there were no signs of increased arterial tens:on. She has not become pregnant again.

Tetany always occurs after some cause producing ex- haustion or defective nutrition of the nervous system. Especially is this the case in the easily-exhausted nervous system of children, in whom tetany is so common as the so-called "carpo-pedal contractions." These contractions may follow convulsions, diarrhcea, teething or any exhausting cause. In adults tetany principally occurs in women, especially from the exhaustion of lactation or pregnancy under unhealthy surroundings ; and also in connexion with hysteria as in a case reported by Dr. Caiger. ${ }^{1}$ Tetany may be regarded as the result of profound exhaustion or malnutrition of the higher motor centres of the cerebral cortex, in consequence of which the restraining influence is with drawn from a lower stratum of cells, which are thus allowed to come into action without any regulation from higher centres. The normal action of this lower stratum of cells is to initiate movements corresponding to the typical attitudes of this disease, consequently when the higher control is withdrawn their uncontrolled action produces permanent contractures. In the case in point the exhaustion of the higher centres was due to the repeated conrulsions, and its intensity was marked by the long period of unconsciousness and absence of voluntary movement; also by the persistent high temperature, which latter is also probably due to the unregulated action of lower heat-producing centres, owing to the exhaustion of higher controlling ones. That no organic lesions exist in tetany is proved by the fact that it may recur in the same subject again and again at intervals. The cardiac murmurs were undoubtedly due to anæmia, although at the time they gave rise to a suspicion of ulcerative endocarditis. 'This is frequently seen as the result of septicamia after delivery, to which cases of eclampsia are particularly exposed owing to the failure of antisepsis in patients with convulsions and suffering from involuntary evacuations. Another point of interest is the increase in the amount of urine and urea which followed measures for the relief of kidney congestion. No treatment was ordered for the tetany except quinine and iron; it is essentially a symptom of deficientaction of the nervous centres and requires a tonic treatment. Dr. Dakin has collected eight cases of tetany curing pregnancy ${ }^{2}$ t they all occurred before deliverynot after, as in the present case-and one ended fatally. Clapham-common, s.W.

\section{A CASE OF TRAUMATIC ANEURYSM OF THE POPLITEAL ARTERY:}

LIGATURE OF ARTERY ABOVE AND BELOW THE WOUND, HOLLOWED BY GANGRENE AND SECONDARY FAEMORRHAGE; AMPUTATION ; RECOVERY.

BY BRIGADE-SURGEON-LIELTENANT-COLONEL C. E. HARRISON, M.B. LoND., F.R.C.S. ENG., GRENADIER GLARDS.

A CORPORAL in the Grenadier Guards was admitted to the Guards Hospital on the evening of October 6th, 1830, with a small punctured wound in the upper third of the calf of the left leg. He stated that in climbing over some iron railings the pointed end of one of the railings had wounded the leg, that he had lost a good deal of blood at the time, and that an officer's servant, who was close by, assisted him into a cab and brought him to the hospital at once. On admission there was little or no bleeding, and on careful examination no evidence could be found that any large vessel was injured. The wound was dressed with carbolic lotion, and the man was ordered to remain in bed. Everything appeared to progress favourably until the $13 \mathrm{th}$, when he stated that in the early morning he had felt his leg uncomfortable. On examining the leg distinct pulsation could be felt and seen over a small area immediately above the level of the wound. There was a little resistance to pressure with the fingers here, but there was no marked swelling. The pulsation was completely controlled by pressure on the femoral artery at the groin. 'The man's urine was found to be free from albumen, but a marked hardness was observed in the radial arteries, which could be traced far up the forearms. The opinion was formed that a small tranmatic aneurysm existed, and the linited 
area and superficial character of the pulsation led to the hope that one of the muscular branches to the gastrocnemius had been wounded rather than the main artery of the limb. It was determined to make an exploratory incision with a view to tying the wounded vessel. A tourniquet having been placed over the femoral artery and an elastic bandage tied firmly round the limb above and below the knee, a probe was carefully introduced into the wound. No reliable information could be obtained by the probe as to the original course of the wound, but it passed rather deeply in an upward direction towards the centre of the popliteal space. An incision, which was subsequently enlarged so as to extend from four inches and a half to five inches, was made from the wound directly over the area of pulsation. The fascia having been divided, the fibres of the head of the gastrocnemius were carefully cut through and the popliteal vein exposed. The tourniquet and elastic bandages having been loosened, the condition of the artery was carefully examined with the finger-without separating it from the vein-both above the level of the gastrocnemius and where covered by the head of that muscle. No extravasated blood was seen, no hæmorrhage occurred, except a very trifling amount from the vessels divided at the time of the operation, and no abnormal condition of the main artery or its branches could be detected. It was not thought advisable to carry the dissection further. A drainage-tube was placed in the lower angle of the wound, which was washed out with a weak solution of perchloride of mercury and closed with silver wire and silk sutures. Antiseptic dressings were applied and the knee was slightly flexed and placed on a splint. The temperature at night was normal. A morphia draught was given. On the following day (14th) the temperature was $99^{\circ}$ in the morning and evening with a pulse of 84 and 88 respectively. On the 15 th the wound was dressed and was looking healthy. There was a little codema about the ankle and leg. The pulsation was found to be quite as marked as before the exploratory operation. In the evening $\mathrm{Mr}$. Howard Marsh kindly saw the patient. He did not consider it possible at that time to express a decided opinion as to the exact injury, but thought the evidence pointed to the wounding of an artery at the time of the accident or to a strain or bruising of the coats of the artery which had subsequently given way. Ho recommended that pressure should be applied to the femoral artery and the condition of the leg watched. The morning temperature was $99^{\circ}$ and pulse 79 . The evening temperature was normal. On the 16 th the leg had been uneasy and a morphia draught had not secured a good night's rest. The wound looked healthy, but there was more swelling on the inner and anterior aspect of the leg. Pulsation could be felt over a rather wider area. There was some discolouration-as from bruising - even as low as the ankle. The temperature was normal ; the pulse 67. In the afternoon pressure was applied over the femoral artery by means of the fingers aided by a sandbag suspended by a cord passed over a pulley screwed into a beam in the ceiling, and this was varied by pressure applied by means of a Skey's tourniquet. The evening temperature was normal and the pulse 64 . On the 18 th the codema was slowly decreasing, but not so the pulsation. A thrill could be felt just on the outer side of the incision and a double bruit heard on auscultation over the area of pulsation. There was some increase of swelling on the inner surface of the calf in its upper part and pulsation was now felt here, and markedly so over the line of the incision. The evening temperature was raised one degree. The pressure was omitted during some part of the night. On the 21st the odema of the limb was less. The pressure had been omitted during the last three nights, but had been continued during the daytime. On the 23rd a continuous thrill, occupying the dilatation and subsidence of the arterial pulse, could be felt on lightly touching the skin over a limited area just to the outer side of the scar left by the incision. Pulsation was marked over the centre of the scar, and could be felt less distinctly up to the posterior border of the tibia on the inner side of the calf. A loud, prolonged, almost continuous musical bruit was audible over the area of pulsation. Pressure had been omitted on the 21st, but resumed after midday on the 22nd. Mr. Marsh again kindly saw the patient and it was determined to try the effect of pressure applied to the limb by means of a perforated Martin's bandage, the foot being kept well raised. It was thought that the local signs indicated the presence of a varicose aneurysm of the posterior tilial or of the popliteal artery just before its division. On the 30th little or no change in the condition of the leg had taken place except that the codema of the limb had been reduced by the bandage. The temperature and pulse were favourable. On Nov. 3rd the aneurysm appeared fuller and tenser. On the 7 th it was felt that some operative interference should be resorted to in order to prevent the risk of rupture of the aneurysm and consequent extravasation into the limb. The aneurysm felt tenser, and the area of pulsation was slowly extending. Mr. Marsh kindly came to the hospital and gave his valuable assistance and advice in the operation. The limb having been raised and an Esmarch's round elastic bandage applied just below the centre of the thigh, an incision was made commencing a little to the inner side of the middle of the popliteal space and continued downwards over the gastrocnemius for about five inches. After carefully cutting through the gastrocnemius and ligaturing those branches of the sural arteries which were divided, a very thin-walled sac was exposed, evidently the sac of the aneurysm. It was thought advisable to expose and ligature the artery above and below the sac. The incision having been extended a little upwards, the tissues were dissected through until the popliteal artery was exposed above the upper boundary of the sac, about the level of the centre of the popliteal space. A double ligature of silk thread, which had previously been boiled in carbolic solution, was applied and the artery cut through between the two ligatures. The dissection was ther continued over the lower end of the sac, the cutaneous incision being extended to the centre of the calf. The upper border of the soleus muscle was exposed, forming the lower boundary of the aneurysmal sac. The fibres of the soleus just below its origin were therefore carefully divided until the posterior tibial nerve was exposed, when the posterior tibial artery was readily found and ligatured. The aneurysmal sac was then incised and opened up nearly to its full extent with scissors. It was then seen that the popliteal vessels bad been pushed towards the outer side of the limb by the aneurysm, and, after the blood and blood-clot had mostly been removed, a round opening, the size of a millet seed, was found on the inner side of the popliteal artery, communicating directly with the sac of the aneurysm about the centre of its outer side. The artery was ligatured above and below thi opening in its walls. The aneurysm had extended deeply in the direction of the interosseous membrane as well as toward the surface of the calf. The Esmarch's bandage having been loosened, the bleeding points were secured. Some dense portions of the clot which were adherent rather firmly to the sac were left in situ. There was a little oozing from the upper extremity of the sac which it was found difficult to arrest. The wound and interior of the sac were sponged out with perchloride of mercury solution, a drainase-tube was inserted into the lower angle of the wound and the part dusted over with iodoform. The wound, which was about nine inches long, was closed with silver wire sutures, and lint moistened with perchloride of mercury solution was applied to the surface. A piece of protective was placed over this and iodoform wool added to cover the whole. A poroplastic back-splint was placed behind the knee and the limb enveloped in cotton-wool. The patient was very chilled after the operation. Hot-water bottles were placed in the bed. In the evening the temperature was $101.2^{\circ}$ and pulse 120. He felt fairly comfortable. The toes of the left foot were cold; there was some slight degree of warmth on the upper surface of the foot. On the 8th the temperature was $102 \cdot 8^{\circ}$; the pulse 128 . He had passed a restless night in conse quence of pain of a burning character in the calf and in the toes which opium had not relieved. The evening temperature was 104.2 and the pulse 150 . The next day the wound was dressed. There had been considerable oozing of blood-stained fluid into the dressings. There was a little fulness to the inner side of the lower part of the wound, which was otherwise looking; well. The drainage-tube was syringed out with perchloride solution and the wound dressed as before. There was staining of the front of the foot and lower part of the front of the leg from extravasation of blood into the superficial part of the skin. The temperature was $1036^{\circ}$, the pulse 139 . The man's aspect was good, and he had passed a fair night. The evening temperature was $1042^{\circ}$, and the pulse 141 . On the 10th some blood-stained fluid had during the night soaked through the lower part of the dressings, and on dressing the wound a gangrenous odour was very marked. The upper part of the foot was rather dusky and mottled-the toes were chilly and the dorsum of the foot and leg were warm. A large bulla - containing dark, blood-stained fluid - hac formed over a considerable area of the lower and inner part of the left leg. The leg up to the knee was infiltrated 
with cedema, and in places there was a blush on the surface of the skin. A dirty, foul-smelling discharge had come from the wound. The temperature was $1036^{\circ}$ and the pulse 125. The advisability of amputating the limb above the knee was discussed ; but, although the man's aspect was good, it was considered that the tempernture and pulse were not favourable for immediate amputation. Mr. Marsh kindly saw the man and recommended that some of the sutures should be removed, the wound freely irrigated and the limb put up in boracic poultices covered with protective and wool. On the 12 th the temperature was $1014^{\circ}$, the pulse 113 . There was less redness of the leg below the wound, which was in a sloughy condition. The wound was irrigated three times during the day with carbolic lotion ( 1 in 80 ) with a little tincture of iodine. The patient was taking food very fairly, with four ounces of brandy, and two grains of quinine three times a day. The evening temperature was $103.2^{\circ}$ and the pulse 114. The opium was continued at night. On the 16 th the temperature had fallen to $98.7^{\circ}$ and the pulse to 96 . The evening temperature was $100 \cdot 8^{\circ}$ and the pulse 104 . On the 17th the wound was looking healthier, but there was a large patch of gangrenous skin and subcutaneous tissue extending over nearly the lower half of the inner side of the leg, and passing round the back and a portion of the outer side. The toes were becoming mummified, and the condition of the foot gave rise to anxiety. The superficial parts on the inner side of the heel were dead, and there was some duskiness with odema of the dorsum, with little or no sensation from just below the ankle in front. The irrigation was continued, a charcoal poultice applied to the lower part of the leg and the brandy increased to six ounces. On the 20 th the temperature was $99.6^{\circ}$ and the pulse 98 . A considerable portion of the soft tissues of the leg and foot was evidently dead, including the greater part of the gastrocnemius and soleus. Mr. Marsh saw the patient, and it was decided to wait and watch from day to day in the hope that the structures of the upper part of the leg would sufficiently recover to justify amputation below the knee. In the event of secondary hæmorrhage occurring it was decided that the limb should be amputated at once. On the 21st secondary hæmorrhage occurred at midday from a point deeply situated in the upper angle of the wound, this being the fifteenth day since the ligatures were applied. The femoral artery was compressed with the fingers and preparation made at once to amputate above the knee. Chloroform having been administered, the limb was removed, anterior and posterior skin flaps being made with circular incision through the muscles. Surgeon-Major Magill, Coldstream Guards, kindly assisted me. Very marked collapse followed, but the patient rallied satisfactorily. The cut surface of the stump was freely washed with perchloride of mercury solution and dressed with strips of gauze moistened with this solution, iodoform having been previously dusted over the skin and edges of the flaps. Catgut ligatures were used and the stump enveloped in sal alembroth wool.

On examining the leg which had been amputated the site from which the hroiorrhage had come appeared to be the point at which the highest ligature had been placed on the popliteal artery. The ligature was almost completely detached and the proximal end of the artery on its inner side looked eroded. There was no evidence of organised clot in this part of the artery. The superior external articular artery was given off a little above this level. The ligature on the popliteal artery immediately below the point where the artery had been cut through after being ligatured was still firmly attached. The walls of the artery in contact with what had been the sac of the aneurysm were partially disorganised. The ligature on the posterior tibial artery was still firmly attached, with a clot above and below. The anterior tibial artery was given off a short distance above this ligature. All the muscles on the back of the leg below the lines of demarcation were in a state of gangrene and in the lower part of the leg the bones were denuded of periosteum. 'The muscles of the sole of the foot were gangrenous and the tissues on the dorsum were becoming so. The patient made a rather slow but a very satisfactory recorery, and was erentually discharged from the service on July 3rd, 1891, with a very useful stump and wearing an artificial limb.

Remarks. - The nature and rarity of the accident in this case render it one of considerable interest. Anxiety as to the best method of treating the case was occasioned by the fact that the physical signs and symptoms left great doubt as to whether the artery alone was wounded or whether a wound of both the artery and the rein had led to a communication between those ressels and the formation of a varicose aneurysm. Under these circumstances it was determined to try the effect of pressure, and, if this failed, to cut down upon the ressels at the seat of injury rather than to apply a ligature higher up. A case in which the symptoms were almost identical with those in the present instance, and in which Mr. Thomas Smith was successful in effecting a cure by means of pressure applied to the limb by a Martin's bandage, led me to hope that a similar good result would be attained in this case. A cure, however, by these means was not attained. The occurrence of sepsis, notwithstanding the care which was taken to prevent this result, must probably be explained by the extent and nature of the wound, which rendered aseptic treatment difficult under the circumstances. The published cases of traumatic aneurysm of the popliteal artery appear to be few in number.

Rochester-row, S.W.

\section{A CASE OF INFLUENZA WITH INTERESTING SEQUELA.}

By LOUIS E. STEVENSON, M.B., B.C. CantaB., LATE HOUSE-SURGEON TO THE CUMBERLAND INFIRMARY, CARLISLE.

THIs case occurred in the practice of my friend, Dr. Stewart Lockie of Carlisle, and to him I am indebted for the following notes. The case had a fatal termination, and at Dr. Lockie's request I conducted the necropsy with him.

A housekeeper aged fifty-seven first came under Dr. Lockie's care on Jan. 25th, 1890 Before this date her health had been impaired for some months, and she particularly suffered from occasional abdominal pain. On Jan. 13th she was seized with a mild attack of influenza, then epidemic in the city. She was convalescent from this and was doing fairly well, but on Jan. 24th she was attacked with severe pain in the abdomen, referred to the hypochondriac, umbilical and epigastric regions. When Dr. Lockie saw her on the 25 th the abdomen was soft, there was a degree of tenderness on pressure, the abdominal muscles moved on respiration, and the pain was not increased on coughing; there was no vomiting; the bowels had been regular; pulse-rate and temperature were normal. A mixture containing sulphate of magnesia and Dover's powder was ordered. On the 27 th there had been some vomiting the bowels had been moved repeatedly and the stools were loose. The pain was unrelieved and had extended low down in the umbilical region and to both lumbar regions. Tenderness was present, but no tumidity. The abdominal muscles still moved in respiration. On the 28 th there was less pain, but on the following day it became much worse and there was some tumidity. There was abdominal tenderness, especially in the right lumbar region; vomiting had ceased. Opium, which had been given at the beginning, was now freely administered with some relief. On the 30 th and 31 st the patient was easier, but the temperature rose to $100^{\circ}$ and the pulse had quickened. Tenderness was now most marked in the left iliac region. In the evening of the 31st it was noticed that the pulse in the right wrist was much more feeble than in the left; only some beats could be felt in the former, whilst in the latter there was a fair regular pulse of 120 . The patient said that for two days the right arm had been slightly numb. At midnight the arm became very painful, cold, and apparently bloodless as far as the elbow. At 3 A.M. on Feb. 1st no pulse could be felt in the right superior extremity below the axilla; the forearm and hand were cold and somerwhat shrivelled, and the hand livid; sensibility was abolished below a line one inch above the wrist. Opium was still more freely administered, and with relief. At this time the pain in the abdomen was less and the tenderness was confined to the left iliac region. There was regurgitation from the stomach, but no actual vomiting. Later on the same day a copious loose stool was passed in which were some specks of blood and shreds of mucus. On the morning of the 2 nd the condition was practically unchanged, but the pulse had disappeared in the axilla. On this day the patient had another copious loose evacuation, but it contained no blood. On the evening of the 2 nd a great change for the worse took place. The face was pinched, the extremities were cold, respiration laboured, and the pulse had risen to 144, being rery feeble. Death took place on the 3rd, at 1.30 A.M.

The necropsy, which was conducted on the 4 th, revealed the following interesting pathological conditions. The abdomen contained a small quantity of black, blood-stained fluid; 